\title{
The satellite communication link establishment scheme for offshore mobile platform
}

\author{
DING YuanMing ${ }^{1,}$, WANG ShiGuang ${ }^{2}$ and SONG Bo ${ }^{2}$ \\ ${ }^{1}$ Network Technology Engineering Laboratory of Smart Shipping and Logistics \\ ${ }^{2}$ Key Laboratory of Communication and Networks, Dalian University \\ No.10, Xuefu Street, JinZhou Xinqu, Dalian 116622, P. R. China \\ adingyuanming@dlu.edu.cn
}

Keywords: Communication on the move; Sea wave model; Link establishment; Mobile platform

\begin{abstract}
Due to the establishment of current maritime satellite communication link is often used communication on the move system, but it often does not apply to the offshore mobile small platform, and the current research in this area is less. This article first on the basis of dynamic simulation of the motion waves by the characteristics analysis of mobile offshore modeling, and then to derive a reasonable window of communication links between satellite communication and mobile offshore platforms. The window for the $2 / 3$ wave period, and may be carried out to establish a link within $1 / 3$ cycle intervals. Finally, according to conclusions link establishment process, which is broadly divided into several modules: initialization information, call connection, establish a connection, send a message, and disconnect the connection.
\end{abstract}

\section{Introduction}

Satellite communication is based on the sight of the communication mode, the request of the communication signal can be transmitted to the satellite receiver, so that the satellite communication terminal at work can be achieved point to point communication mode [1, 2]. After the study found that the sea has a relatively complex channel environment, offshore mobile platform at any time will be affected by the sea waves and other adverse factors. Therefore, the mobile small platform at sea is extremely unstable. In bad sea conditions, the amplitude of swing of the small offshore mobile platform will be very large, and therefore lead to satellite communication links illegal interruption caused by data transmission interrupt, the important transmission data will be lost, which makes the data packet loss rate increased, and caused great losses. If we want to make a stable communication between the small platform and the satellite, we must design a link to establish a stable performance, so as to make the performance of communication greatly improved. The process of offshore mobile platform and satellite communication can be used receiving antenna of small servo system, and enhance the satellite signal receiving device, etc., but these methods are not suitable for offshore mobile platform, so you can use wave cycle and small mobile offshore platform motion characteristics, choose reasonable satellite communication time, unfavorable factors into favorable factors, greatly reducing the offshore mobile platform of small satellite communication data packet loss rate[3, 4].

\section{Dynamic Ocean Wave}

Because of the marine environment is very complex, state of mobile platforms at sea have a crucial influence, so we must through the simulation of the dynamic wave. For maritime mobile satellite link establishment conditions.

Dynamic wave spectrum is the Fourier transform of the sea surface height fluctuation function, through the wave spectrum can be reflect the sea plane harmonic components with respect to the space and the location of the distribution, to understand the real waves must carries on the simulation. 
Moscowitz and Pierson through investigation of the North Pacific Sea, obtains the dimensionless spectrum (P-M spectrum $[5,6])$. Its expression is as follows:

$$
S(\omega)=\frac{a g^{2}}{\omega^{5}} \exp \left[-\gamma\left(\frac{g}{U_{19.5} \omega}\right)^{4}\right] .
$$

Among them, the wave spectrum value of $S(\omega)$ for $\omega, \omega(\mathrm{rad} / \mathrm{s})$ is the spatial frequency of the waves; $U_{19.5}$ is wind speed for the distance from the sea 19.5 high; The other parameters of the values, $a \approx 0.0081$, the experience constant $\gamma=0.74$, gravity acceleration $g=9.8 \mathrm{~m} / \mathrm{s}^{2}$. Finally, according to the dispersion relation $s(k)=s(\omega) d \omega / d t$. The two-dimensional P-M spectrum of the expression is as follows:

$$
S(k, \varphi)=\frac{a}{4 k^{4}} \exp \left(-\frac{r g^{2}}{k^{2} U_{19.5}}\right) \cos ^{4}\left(\frac{\varphi-\varphi_{m}}{2}\right) .
$$

The $\varphi$ in the formula is a two-dimensional P-M spectrum. In the xoy plane, the angle formed between the counter clockwise direction of the $x$ axis and the direction of the aim. $\varphi_{m}$ is the direction of wind speed. Through Monte Carlo simulation method canobtain the 4 and 6 level sea waves of P-M spectrum. Simulation results show that, with sea wave level increase, sea wave lengths will increase correspondingly and wave height will be increased. The model can effectively reflect the different sea real marine environment, for maritime mobile satellite communication link is established to provide the preparation conditions.

\section{Motion Characteristics of the Offshore Mobile Platform}

In the maritime mobile small platforms may produce the six degree of freedom movement, rising and sinking, heave and roll is in resilience or righting moment function under, have to balance the location for the periodicity of transverse wave and heave motion.

Heaving Motion of a Small Platform on the Sea. With reference to the horizontal plane, we choose the time when the mobile platform and the sea level are both static. Then find what position of waterline mobile small platform on the sea. According to the summary of the previous chapters is known,we can use standard cosine to approximate the irregular waves, then we can be defined the vertical distand from waterline to reference horizontal plane as $\mathrm{x}$; The vertical distance between the surface of the ocean wave and the reference plane is defined as $y$, in which the wave of the simulated cosine formula $y=\operatorname{ros} \omega t$; The vertical distance from the reference plane to any point below the sea is what we call z; In Fig. 1, x, y, z direction is down to the positive [7].

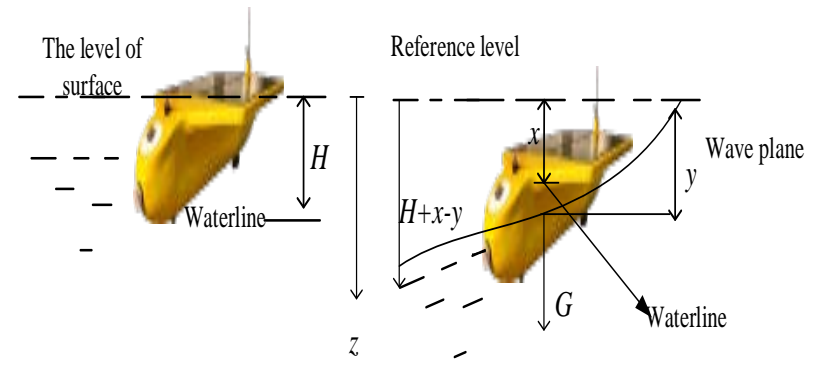

Fig.1. The motion of a small platform on the sea by the waves.

$$
\left(m+m^{\prime}\right) \ddot{x}+b \dot{x}+5 \rho g A x=r e^{-H K}\left[\left(5 \rho g A-M^{\prime} \omega^{2}\right) \cos \omega t-b \omega \sin \omega t\right] .
$$

where, $c=5 \rho g A$ as recovery constant; $m_{v}=m+m$ as virtual quality.

Set up $2 n=b / m_{v}$ and $\omega_{n}^{2}=c / m_{v}$, can be obtained:

$$
x+\ddot{2} n \dot{x}+\omega_{n}^{2} x=\frac{F_{0}}{m_{v}} \cos (\omega t+\theta)
$$


where, $F_{0}=r e^{-H K} \sqrt{\left(c-m^{\prime} \omega^{2}\right)^{2}+b^{2} \omega^{2}}$ is the excitation force, $\theta=\tan ^{-1}\left(-b \omega /\left(c-m^{\prime} \omega^{2}\right)\right)$ is the angle between the excitation force and the sea wave.

According to the above general equations of motion, the special solution is

$$
x(t)=\frac{F_{0}}{m_{v} \sqrt{\left(\omega_{n}^{2}-\omega^{2}\right)^{2}+4 n^{2} \omega^{2}}} \cos (\omega t+\theta+\varphi) .
$$

$\varphi=\tan ^{-1}\left(-2 n \omega /\left(\omega_{n}^{2}-\omega^{2}\right)\right)$ is the phase angle of between the excitation force and heave motion. From this we can draw the change curve of the vertical swing of the small platform on the sea with time as shown in Fig. 2.

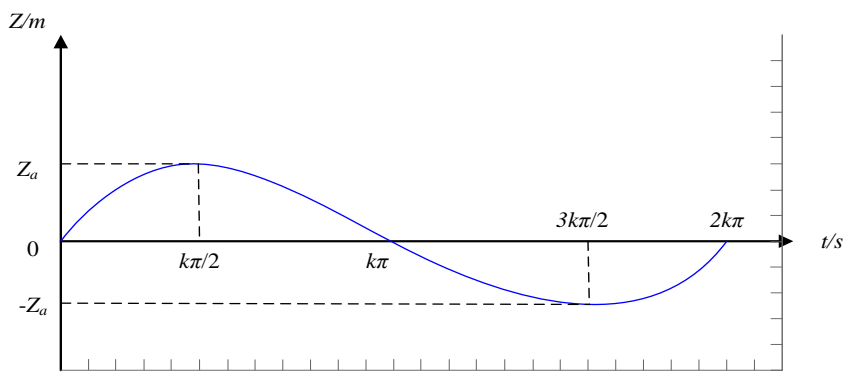

Fig.2 The change curve of the vertical swing of the small platform on the sea

Rolling Motion of a Small Platform on the Sea. Offshore mobile platform with satellite communication in the complex ocean environment may occur offshore mobile platform dumping situation, due to the influence by the bad sea environment. In order to make offshore mobile platform with satellite normal and steady communication in the water, meanwhile overcome the maritime bad environment does not happen dumping, so the small platform must be designed to ensure the stability.

Figure 3 is a transient state, the state is maritime mobile platform instantaneous state of the roll motion in the waves, in Fig. $3 \delta$ is inclination angle, $\alpha$ is the angle between the wave, $G$ is the mobile offshore platform of center of gravity, $F$ is the center of buoyancy, $K$ is the force of gravity,it throughs the center of gravity $G, L$ is the buoyancy, it throughs the center of buoyancy $F$, they are vertical to the wave plane. In Fig. 3, wind resistance $f_{1}$ act on $W$, seawater resistance $f_{2}$ act on $V$, water flow direction and wind flow direction are parallel to sea wave .

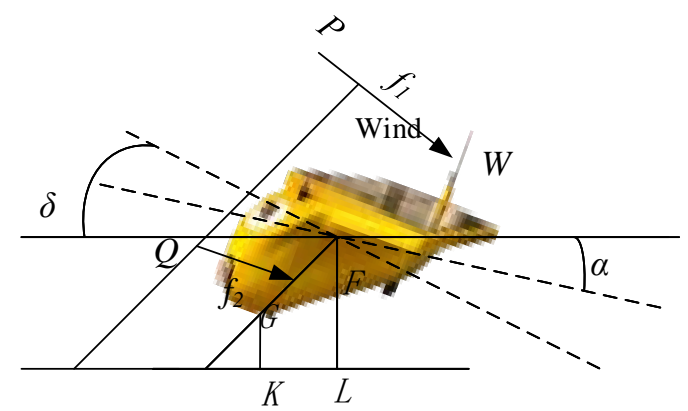

Fig.3 Sea mobile platform with the wave of the wave is the attitude

As shown in Fig. 3, Balance equation can be established:

$$
\begin{aligned}
J_{\varphi} \ddot{\varphi}= & -\left(M_{G}+M_{f 1}+M_{F 2}\right)=-\left(D \times \overline{K F}+f_{1} \times \overline{F P}+f_{2} \times \overline{F Q}\right) \\
& =-\left(D \times \overline{G F} \sin (\varphi-\alpha)+\left(f_{1} \times \overline{F W}+f_{2} \times \overline{F V}\right) \cos (\varphi-\alpha)\right) .
\end{aligned}
$$

From this we can draw the curve of the horizontal swing of the small platform on the sea with time as shown in Fig. 4.

According to the above formula is derived and shown in Fig.2 and Fig.4 , we found small mobile offshore platform in the cycle of a wave is regular heave, and about a period of a quarter in the trough, the most important information provided here is wave cycle. This gives us some useful assumptions about the character of the wave shadow fading signal. If the wave period is 5 to 10 seconds, then we can infer that the offshore mobile small platform will have 1/3 cycle or 1 to 3 seconds in the trough. The duration of the signal loss will depend on the elevation of the satellite and the height of the 
antenna from the surface of the sea. As a result, we must be able to recover from a signal loss period of 1 to 3 seconds.

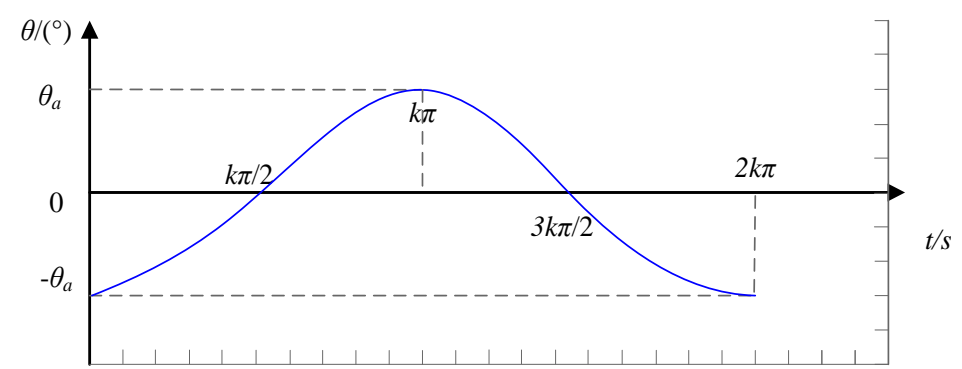

Fig.4 The transformation curve of the horizontal swing of the small platform on the sea

\section{Link Establishment Scheme}

The communication link is established on the segment, and the two sides can carry out data transmission on the channel. The process of establishing the single link between the satellite and the small platform node is generally the following process:

Maritime mobile communication link is established between the small platform nodes $X$ and satellite node $\mathrm{Y}$, node $\mathrm{X}$ will sends link establishment request information to node $\mathrm{Y}$, when after the node $\mathrm{Y}$ receives the link request from node $\mathrm{X}$, and node $\mathrm{Y}$ sends a confirmation connection signal to node $\mathrm{X}$, when node $\mathrm{X}$ receives the confirmation signal from node $\mathrm{Y}$, which demonstrate the link completely setup, you can carry out normal communication.

Link establishment scheme is divided into several modules: Initialization information, Call connection, Establish connection, Send message and Disconnect connection. Specific link establishment process is as follows:

Step1: according to a set of good communication time window, in the communication window to start the link of the antenna link function, the itself information and to link the establishment of the request information package, sent to the satellite receiving terminal. If the connection fails, the link is in the closed state to check whether it is still in the window, and then continue to send the request link to establish the request signal.

Step2: when the sending complete to send the link establishment request signal to wait for a connection confirmation signal, the satellite will send confirmed signal to the sender, the sender receives an acknowledgement signal, show that the link was established successfully. If there is no overtime and no acknowledgement signal is received in which waits for the confirmation phase, then wait until timeout to close the link state, then repeat Step1.

Step3: after link establishment successful,then enter the formal link communication phase (timing sending data), according to the characteristics of wave cycle and mobile offshore platform sagging swings of the cycle, there will be $3 / 4$ period for sending time, if data transmission is finished or the sending time window is closed,the link is closed state and wait for the next transmission time window is open, and then repeat Step1.

Step4: satellite receiver receives information, when satellite receives information from mobile offshore platform and sends a reply signal to the sender, when the receiver receives a reply signal that send information completed. If you do not receive the corresponding reply signal within the specified time and the link still is the sending window, the data will be retransmitted. The 3 time retransmission is still no answer to exit the current communication link and recreate a new link, and then wait for the next time window to open, and then repeat Step1. 


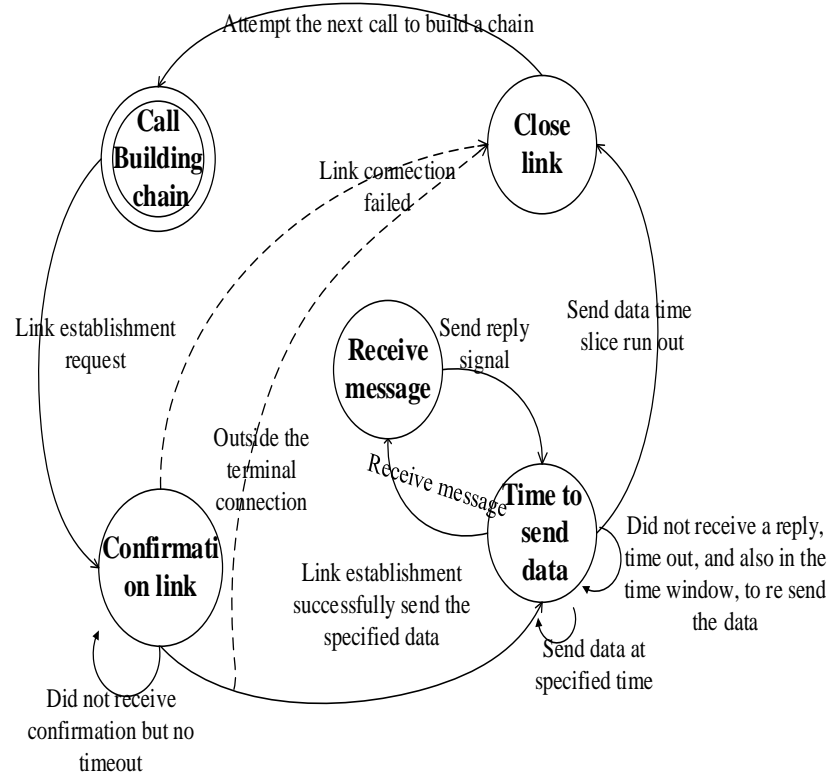

Fig.5. Link establishment procedure

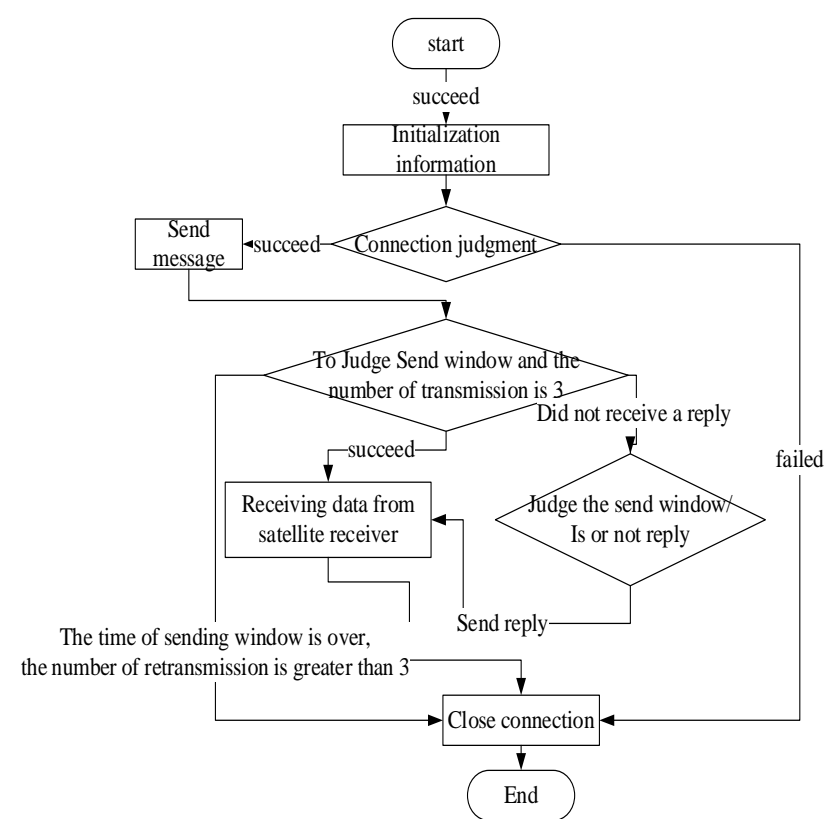

Fig.6. Link establishment flow chart

\section{Summary}

By the dynamic simulation of ocean waves obtain wave period, the sea state and related to waves information, then through analysis of the motion characteristics of the mobile offshore platform, and then analysis of the waves of vertical swing and rolling motion effect on the motion state of the mobile offshore platform. At last, according to the cycle of ocean waves and the movement of the small platform obtain the establishment plan of the communication link. Link establishment scheme is divided into several modules: Initialization information, Call connection, Establish connection, Send message and Disconnect connection.

\section{Acknowledgements}

This work was financially supported by the National Natural Science Foundation of China (61540024) and the Program for Liaoning Innovative Research Team in University（LT2014025）.

\section{References}

[1] X. Hongyan, W. Mingming and F. Yumin: Selecting prediction model for sea mobile communication. Journal of Beijing Jiaotong University. Vol. 29 (2012), p. 65.

[2] K. Xuehai. K. Shuren: Pseudo mono pulse auto tracking antenna system for Ku-band mobile satellite communication terminal. Communication and Information technology. Vol. 17 (2010), p. 17.

[3] H.L. Yee, D. Feng and S.M. Yu: Near sea-surface mobile radio wave propagation at 5 GHz: Measurements and modeling. Radio Engineering, Vol. 824 (2014), p. 23.

[4] A.M. Saegh, A. Sali and J.S. Mandeep: Atmospheric propagation model for satellite communications. MATLAB Applications for the Practical Engineer (2014).

[5] Z. Li, W. Haitao and Z. Qiaobin: Research on dynamic simulation of seas. Ship Electronic Engineering. Vol. 35 (2013), p. 82.

[6] Z. Jianwu, Z. Mingbo and D. Wei: An overview of ocean wave modeling. Ship Electronic Engineering. Vol. 11 (2010), p. 10.

[7] L. Dianpu: Study of ship motion modeling and prediction based on periodogram methods. Ship engineering (2013). 